COMMUNICATION

\title{
3-Halotetrahydropyran-4-one derivatives from homopropargyl acetal
}

Jon Erik Aaseng, Naseem Iqbal, Jørn Eivind Tungen, Christian A. Sperger and Anne Fiksdahl*

Department of Chemistry, Norwegian University of Science and Technology, NTNU, Høgskoleringen, NO-7491 Trondheim, Norway

Corresponding author: Anne Fiksdahl; E-mail: anne.fiksdahl@chem.ntnu.no; ph: (+47) 73594094

\section{Supplementary Information}




\section{Supplementary Information}

Full experimental details, ${ }^{1} \mathrm{H}$ and ${ }^{13} \mathrm{C}$ NMR, IR and HRMS characterization of compounds 8, 9, 10, 11, 12a, 12b, 13 and 14 and copies of ${ }^{1} \mathrm{H}$ and ${ }^{13} \mathrm{C}$ NMR spectra of compounds $7-14$.

\section{3-Bromo-4-methoxy-6-(4-methoxyphenyl)-2,2-dimethyl-3,6-dihydro-2H-pyran (8)}

Gold catalyst $1(12.1 \mathrm{mg}, 5 \mathrm{~mol} \%)$ in dry nitromethane $(2.0 \mathrm{ml})$ was added a solution of acetal $2^{[1]}(79$ $\mathrm{mg}, 0.32 \mathrm{mmol})$ in nitromethane $(2.0 \mathrm{ml})$ and stirred for $3 \mathrm{~min}$. NBS (58 $\mathrm{mg}, 1$ equiv.) was added in one portion and the reaction continued for $15 \mathrm{~min}$. Dichloromethane $(20 \mathrm{ml})$ and a solution of $\mathrm{NaHCO}_{3}$ (saturated, $10 \mathrm{ml}$ ) were added. The aqueous phase was extracted with additional dichloromethane $(10 \mathrm{ml})$ and the combined organic phases were dried over $\mathrm{MgSO}_{4}$. The concentrated crude material was purified flash (VersaFlash) chromatography (2\% EtOAc in $n$-pentane) to give 8 (52 mg, 50\%) as a colourless oil; $\mathrm{R}_{f}=0.18\left(5 \%\right.$ EtOAc in $n$-pentane). ${ }^{1} \mathrm{H}$ NMR (400 MHz, $\left.\mathrm{CDCl}_{3}\right) \delta$ $7.39\left(\mathrm{~d}, J=8.7 \mathrm{~Hz}, 2 \mathrm{H}, \mathrm{H}_{A r}\right), 6.89\left(\mathrm{~d}, J=8.7 \mathrm{~Hz}, 2 \mathrm{H}, \mathrm{H}_{A r}\right), 5.26(\mathrm{br} \mathrm{s}, 1 \mathrm{H}, \mathrm{H}-6), 4.69$ (d, $J=1.9 \mathrm{~Hz}$, 1H, H-5), 4.24 (br s, 1H, H-3), 3.80 (s, 3H, $\left.\mathrm{CH}_{3} \mathrm{OC}_{A r}\right), 3.59$ (s, 3H, $\left.\mathrm{CH}_{3} \mathrm{O}\right), 1.52$ (s, $\left.3 \mathrm{H}, \mathrm{CH}_{3} \mathrm{C}\right), 1.49$ $\left(\mathrm{s}, 3 \mathrm{H}, \mathrm{CH}_{3} \mathrm{C}\right) .{ }^{13} \mathrm{C}$ NMR $\left(100 \mathrm{MHz}, \mathrm{CDCl}_{3}\right) \delta 159.6\left(\mathrm{C}_{A r}\right), 152.4(\mathrm{C}-4), 133.9\left(\mathrm{C}_{A r}\right), 129.0\left(\mathrm{C}_{A r}\right)$, $114.0\left(\mathrm{C}_{A r}\right), 99.0(\mathrm{C}-5), 73.8(\mathrm{C}-2), 72.9(\mathrm{C}-6), 55.3\left(\mathrm{CH}_{3} \mathrm{OC}_{A r}\right), 54.8\left(\mathrm{CH}_{3} \mathrm{O}\right), 54.4(\mathrm{C}-3), 30.0$ $\left(\mathrm{CH}_{3} \mathrm{C}\right), 22.5\left(\mathrm{CH}_{3} \mathrm{C}\right)$. IR (neat, $\left.\mathrm{cm}^{-1}\right) 2336(\mathrm{w}), 1611(\mathrm{~m}), 1512(\mathrm{~s}), 1370(\mathrm{~m}), 1246(\mathrm{~s}), 1172$ (s), $1102(\mathrm{~s}), 1028(\mathrm{~s})$.

\section{3,5-Dibromo-4-methoxy-2-(4-methoxyphenyl)-6,6-dimethyl-3,6-dihydro-2H-pyran (9) and 3,5-}

\section{Dibromo-4-methoxy-6-(4-methoxyphenyl)-2,2-dimethyl-3,6-dihydro-2H-pyran (10)}

The gold catalyst $\mathbf{1}(8.0 \mathrm{mg}, 5 \mathrm{~mol} \%)$ was dissolved in dry nitromethane $(1.0 \mathrm{ml})$ and added a solution of acetal $2^{[1]}(50 \mathrm{mg}, 0.20 \mathrm{mmol})$ in nitromethane $(1.0 \mathrm{ml})$. After $3 \mathrm{~min}$ stirring, NBS $(72 \mathrm{mg}, 2$ equiv.) was added in one portion. The reaction continued for $15 \mathrm{~min}$ before addition of a solution of $\mathrm{NaHCO}_{3}$ (saturated, $10 \mathrm{ml}$ ) and dichloromethane $(20 \mathrm{ml})$. The aqueous phase was extracted with dichloromethane $(10 \mathrm{ml})$, and the combined organic phases were dried over $\mathrm{MgSO}_{4}$. The concentrated 
crude material was purified through flash (VersaFlash) chromatography (2\% EtOAc in $n$-pentane) to afford $\mathbf{9}(25 \mathrm{mg}, 31 \%)$ and $\mathbf{1 0}(12 \mathrm{mg}, 15 \%)$ as colourless oils.

9: $\mathrm{R}_{f}=0.37\left(5 \%\right.$ EtOAc in $n$-pentane). ${ }^{1} \mathrm{H}$ NMR $\left(400 \mathrm{MHz}, \mathrm{CDCl}_{3}\right) \delta 7.31\left(\mathrm{~d}, J=8.7 \mathrm{~Hz}, 2 \mathrm{H}, \mathrm{H}_{A r}\right)$, $6.92\left(\mathrm{~d}, J=8.9 \mathrm{~Hz}, 2 \mathrm{H}, \mathrm{H}_{A r}\right), 4.93(\mathrm{~d}, J=1.6 \mathrm{~Hz}, 1 \mathrm{H}, \mathrm{H}-2), 4.64(\mathrm{~d}, J=2.0 \mathrm{~Hz}, 1 \mathrm{H}, \mathrm{H}-3), 3.86(\mathrm{~s}, 3 \mathrm{H}$, $\left.\mathrm{CH}_{3} \mathrm{O}\right), 3.82\left(\mathrm{~s}, 3 \mathrm{H}, \mathrm{CH}_{3} \mathrm{OC}_{\mathrm{Ar}}\right), 1.60\left(\mathrm{~s}, 3 \mathrm{H}, \mathrm{CH}_{3} \mathrm{C}\right), 1.50\left(\mathrm{~s}, 3 \mathrm{H}, \mathrm{CH}_{3} \mathrm{C}\right) \cdot{ }^{13} \mathrm{C} \mathrm{NMR}\left(100 \mathrm{MHz}, \mathrm{CDCl}_{3}\right)$ $\delta 159.2\left(\mathrm{C}_{A r}\right), 148.6(\mathrm{C}-4), 130.7\left(\mathrm{C}_{A r}\right), 127.3\left(\mathrm{C}_{A r}\right), 114.5(\mathrm{C}-5), 113.5\left(\mathrm{C}_{A r}\right), 77.9(\mathrm{C}-6), 71.8(\mathrm{C}-2)$, $57.5\left(\mathrm{CH}_{3} \mathrm{OC}_{\mathrm{Ar}}\right), 55.3\left(\mathrm{CH}_{3} \mathrm{O}\right), 50.6(\mathrm{C}-3), 29.3\left(\mathrm{CH}_{3} \mathrm{C}\right), 24.0\left(\mathrm{CH}_{3} \mathrm{C}\right)$. IR (neat, $\left.\mathrm{cm}^{-1}\right) 2935(\mathrm{w}), 1642$ (w), 1614 (w), 1513 (s), 1461 (w), 1305 (m), 1247 (s), 1159 (m), 1095 (m), 1033 (m). HRMS (ESI) calcd for $\mathrm{C}_{15} \mathrm{H}_{18} \mathrm{O}_{3} \mathrm{Br}_{2}\left(\mathrm{M}^{\dagger}\right)$ 403.9617, obsd 403.9618.

10: $\mathrm{R}_{f}=0.29\left(5 \%\right.$ EtOAc in $n$-pentane). ${ }^{1} \mathrm{H}$ NMR $\left(400 \mathrm{MHz}, \mathrm{CDCl}_{3}\right) \delta 7.38\left(\mathrm{~d}, J=8.7 \mathrm{~Hz}, 2 \mathrm{H}, \mathrm{H}_{A r}\right)$, $6.89\left(\mathrm{~d}, J=8.5 \mathrm{~Hz}, 2 \mathrm{H}, \mathrm{H}_{A r}\right), 5.17$ (d, $\left.J=1.1 \mathrm{~Hz}, 1 \mathrm{H}, \mathrm{H}-6\right), 4.49$ (d, $\left.J=1.3 \mathrm{~Hz}, 1 \mathrm{H}, \mathrm{H}-3\right), 3.88$ (s, 3H, $\left.\mathrm{CH}_{3} \mathrm{O}\right), 3.80\left(\mathrm{~s}, 3 \mathrm{H}, \mathrm{CH}_{3} \mathrm{OC}_{\mathrm{Ar}}\right), 1.56\left(\mathrm{~s}, 3 \mathrm{H}, \mathrm{CH}_{3} \mathrm{C}\right), 1.49$ (s, 3H, $\left.\mathrm{CH}_{3} \mathrm{C}\right) .{ }^{13} \mathrm{C} \mathrm{NMR}\left(100 \mathrm{MHz}, \mathrm{CDCl}_{3}\right)$ $\delta 159.9\left(\mathrm{C}_{A r}\right), 149.4(\mathrm{C}-4), 131.5\left(\mathrm{C}_{A r}\right), 130.0\left(\mathrm{C}_{A r}\right), 113.9\left(\mathrm{C}_{A r}\right), 108.0(\mathrm{C}-5), 77.6(\mathrm{C}-6), 74.3(\mathrm{C}-2)$, $57.9\left(\mathrm{CH}_{3} \mathrm{O}\right), 55.2\left(\mathrm{CH}_{3} \mathrm{OC}_{\mathrm{Ar}}\right), 53.9(\mathrm{C}-3), 29.6\left(\mathrm{CH}_{3} \mathrm{C}\right), 22.5\left(\mathrm{CH}_{3} \mathrm{C}\right)$. IR (neat, $\left.\mathrm{cm}^{-1}\right) 2937(\mathrm{w}), 1652$ (w), 1612 (w), 1513 (m), 1461 (w), 1304 (m), 1238 (m), 1173 (m), 1001 (m), 734 (s). HRMS (ESI) calcd for $\mathrm{C}_{15} \mathrm{H}_{18} \mathrm{O}_{3} \mathrm{Br}_{2}\left(\mathrm{M}^{+}\right)$403.9617, obsd 403.9624.

\section{1-(1-Ethoxybut-3-ynyl)-4-methoxybenzene (11)}

The title compound was isolated as the main product in an attempted gold-catalysed cyclization/iodination sequence of homopropargyl acetal 3. ${ }^{[1]}$ Gold-catalyst 1 (8.0 mg, 5 mol\%) was dissolved in DCM (1.0 ml), added NIS (94 mg, 2 equiv.) and stirred for a few minutes. A solution of homopropargyl acetal $3(54 \mathrm{mg}, 0.22 \mathrm{mmol})$ in DCM $(1.0 \mathrm{ml})$ was added over $5 \mathrm{~min}$. A rapid conversion of 3 was observed, and after 15 min the reaction was added triethylamine ( 5 drops), before the mixture was filtered through Celite and concentrated. The residue was purified by flash (VersaFlash) chromatography (3\% EtOAc in $n$-pentane). Compound 11 (18 mg, 41\%) was isolated as a colourless oil; $\mathrm{R}_{f}=0.50$ (3\% EtOAc in $n$-pentane). ${ }^{1} \mathrm{H}$ NMR $\left(400 \mathrm{MHz}, \mathrm{CDCl}_{3}\right) \delta 7.26$ (app d, $J=$ $\left.9.0 \mathrm{~Hz}, 2 \mathrm{H}, \mathrm{H}_{A r}\right), 6.89\left(\operatorname{app} \mathrm{d}, J=9.0 \mathrm{~Hz}, 2 \mathrm{H}, \mathrm{H}_{A r}\right), 4.37$ (t, $\left.J=6.7 \mathrm{~Hz}, 1 \mathrm{H}, \mathrm{H}-1\right), 3.81\left(\mathrm{~s}, 3 \mathrm{H}, \mathrm{CH}_{3} \mathrm{O}\right)$, $3.39\left(\mathrm{dq}, J=7.0,2.0 \mathrm{~Hz}, 2 \mathrm{H}, \mathrm{CH}_{3} \mathrm{CH}_{2}\right), 2.69$ (ddd, $\left.J=16.6,6.6,2.6 \mathrm{~Hz}, 1 \mathrm{H}, \mathrm{H}-2\right), 2.52$ (ddd, $J=$ 
16.2, 6.8, 2.7 Hz, 1H, H-2), $1.95(\mathrm{t}, J=2.6 \mathrm{~Hz}, 1 \mathrm{H}, \mathrm{H}-4), 1.18\left(\mathrm{t}, J=7.0 \mathrm{~Hz}, 3 \mathrm{H}, \mathrm{CH}_{3} \mathrm{CH}_{2}\right) .{ }^{13} \mathrm{C} \mathrm{NMR}$ $\left(100 \mathrm{MHz}, \mathrm{CDCl}_{3}\right) \delta 159.3\left(\mathrm{C}_{A r}\right), 133.2\left(\mathrm{C}_{A r}\right), 127.8\left(\mathrm{C}_{A r}\right), 113.7\left(\mathrm{C}_{A r}\right), 81.1\left(\mathrm{CH}_{2} C_{\text {alkyne }}\right), 79.6$ $\left(\mathrm{HCC}_{A r}\right), 69.8\left(\mathrm{HC}_{\text {alkyne }}\right), 64.2\left(\mathrm{OCH}_{2} \mathrm{CH}_{3}\right), 55.2\left(\mathrm{CH}_{3} \mathrm{OC}_{A r}\right), 28.1\left(\mathrm{CH}_{2} \mathrm{C}_{\text {alkyne }}\right), 15.2\left(\mathrm{CH}_{3} \mathrm{CH}_{2}\right) . \mathrm{IR}$ (neat, $\left.\mathrm{cm}^{-1}\right) 3291(\mathrm{w}), 2973(\mathrm{w}), 1612(\mathrm{~m}), 1511$ (s), 1246 (s), 1173 (m), 1091 (s), 1034 (m), 830 (m). HRMS (ESI) calcd for $\mathrm{C}_{10} \mathrm{H}_{13} \mathrm{O}_{2}\left(\mathrm{M}-\mathrm{C}_{3} \mathrm{H}_{3}\right)^{+}$165.0910, obsd 165.0913 .

\section{General procedure for acid catalysed hydrolysis of dihydropyrans 8 and 9:}

Experiments were performed with TsOH. $\mathrm{H}_{2} \mathrm{O}$, according to the protocol described by Bae and coworkers. ${ }^{[2]}$ Weaker acids, such as CSA and TFA were tested, as well. The amount of acid and the reaction times were also varied in modified protocols.

\section{General procedure for the preparation of esters 12a,b:}

A solution of 1-(4-methoxyphenyl)buta-2,3-dien-1-ol in DCM $(\mathrm{c}=200 \mathrm{mM})$ was added DMAP $(0.05$ equiv.) and $\mathrm{NEt}_{3}$ (2.0 equiv.) at $0{ }^{\circ} \mathrm{C}$ and stirred for $15 \mathrm{~min}$. The acyl chloride or anhydride reactant (3.0 equiv.) was added at $0{ }^{\circ} \mathrm{C}$ and the mixture was stirred at $\mathrm{rt}$ for $20 \mathrm{hrs}$. Diethyl ether $(20 \mathrm{~mL} / \mathrm{mmol}$ alcohol) was added and the solution was treated with $\mathrm{HCl}(1 \mathrm{M}, 20 \mathrm{~mL} / \mathrm{mmol}$ alcohol), brine (20 $\mathrm{mL} / \mathrm{mmol}$ alcohol $)$, aq. $\mathrm{NH}_{3}(6 \%, 2 \times 20 \mathrm{~mL} / \mathrm{mmol}$ alcohol $)$ and brine $(2 \times 15 \mathrm{~mL} / \mathrm{mmol}$ alcohol $)$. The organic phase was dried over $\mathrm{Na}_{2} \mathrm{SO}_{4}$ and concentrated.

\section{1-(4-Metoxyfenyl)-2-metylbuta-2,3-dien-1-yl benzoate (12a).}

The title compound was prepared according to the General procedure above from 1-(4methoxyphenyl)buta-2,3-dien-1-ol (0.24 g, $1.26 \mathrm{mmol})$, DMAP ( $8 \mathrm{mg}, 0.06 \mathrm{mmol}, 0.05$ equiv.), $\mathrm{NEt}_{3}$ (0.26 g, $2.52 \mathrm{mmol}, 2.0$ equiv.) and benzoyl chloride ( $0.53 \mathrm{~g}, 3.78 \mathrm{mmol}, 3.0$ equiv.). Flash chromatography ( $n$-pentane/EtOAc 10:1) afforded 12a $(0.32 \mathrm{~g}, 87 \%)$ as a colourless clear oil; $R_{f}=$ 0.44 (n-hexane/EtOAc 4:1). ${ }^{1} \mathrm{H}$ NMR (400 MHz, $\left.\mathrm{CDCl}_{3}\right) \delta: 8.10-8.08\left(\mathrm{~m}, 2 \mathrm{H}, \mathrm{H}_{A r}\right), 7.56-7.52(\mathrm{~m}, 1$ $\left.\mathrm{H}, \mathrm{H}_{A r}\right), 7.45-7.41\left(\mathrm{~m}, 2 \mathrm{H}, \mathrm{H}_{A r}\right), 7.40-7.38\left(\mathrm{~m}, 2 \mathrm{H}, \mathrm{H}_{A r}\right), 6.90-6.88\left(\mathrm{~m}, 2 \mathrm{H}, \mathrm{H}_{A r}\right), 6.36(\mathrm{t}, J=2.4 \mathrm{~Hz}$, $1 \mathrm{H}, \mathrm{ArCH}), 4.86-4.75\left(\mathrm{~m}, 2 \mathrm{H}, \mathrm{CH}_{2}\right), 3.80\left(\mathrm{~s}, 3 \mathrm{H}, \mathrm{OCH}_{3}\right), 1.69$ (t, $\left.J=3.2 \mathrm{~Hz}, 3 \mathrm{H}, \mathrm{CH}_{3}\right) .{ }^{13} \mathrm{C} \mathrm{NMR}$ $\left(100 \mathrm{MHz}, \mathrm{CDCl}_{3}\right) \delta: 206.4(\mathrm{C}=C=\mathrm{C}), 165.5(\mathrm{COPh}), 159.5\left(\mathrm{CH}_{3} \mathrm{OC} C_{A r}\right), 133.0\left(p-\mathrm{C}_{A r}\right), 130.6$ 
$\left(\mathrm{CHC}_{A r}\right), 130.4\left(\mathrm{O}_{2} \mathrm{CC}_{A r}\right), 129.7\left(2 \times o-\mathrm{C}_{A r}\right), 128.5\left(2 \times o-\mathrm{C}_{\mathrm{Ar}}\right), 128.4\left(2 \times m-\mathrm{C}_{A r}\right), 113.8\left(2 \times m-\mathrm{C}_{A r}\right)$, $99.4\left(C=\mathrm{C}=\mathrm{CH}_{2}\right), 76.9\left(\mathrm{CH}_{2}\right), 76.2(\mathrm{CH}), 55.3\left(\mathrm{OCH}_{3}\right), 15.2\left(\mathrm{CH}_{3}\right)$. IR (neat, $\left.\mathrm{cm}^{-1}\right) 2953(\mathrm{br}), 2836$ (w), 1960 (w), 1716 (s), $1511(\mathrm{~m}), 1450$ (m), 1243 (s), 1116 (m), 707 (s). HRMS (ESI) calcd for $\mathrm{C}_{19} \mathrm{H}_{18} \mathrm{O}_{3} \mathrm{Na}[\mathrm{M}+\mathrm{Na}]^{+}$317.1148, obsd 317.1143.

\section{1-(4-Metoksyfenyl)-2-metylbuta-2,3-dien-1-yl acetate (12b).}

The title compound was prepared according to the General procedure above from 1-(4methoxyphenyl)buta-2,3-dien-1-ol (0.15 g, 0.79 mmol), DMAP (5 mg, 0.04 mmol, 0.05 equiv.), $\mathrm{NEt}_{3}$ (0.16 g, $1.57 \mathrm{mmol}, 2.0$ equiv.) and acetic acid anhydride $(0.24 \mathrm{~g}, 2.36 \mathrm{mmol}, 3.0$ equiv.). Flash chromatography ( $n$-pentane/EtOAc 10:1) afforded product $\mathbf{1 2 b}(0,14 \mathrm{~g}, 76 \%)$ as a yellow oil; $R_{f}=$ 0,41 (n-hexane/EtOAc 4:1). ${ }^{1} \mathrm{H}$ NMR (400 MHz, $\left.\mathrm{CDCl}_{3}\right) \delta$ : 7.32-7.28 (m, $\left.2 \mathrm{H}, \mathrm{H}_{A r}\right), 6.91-6.89$ (m, 2 $\left.\mathrm{H}, \mathrm{H}_{A r}\right), 6.12(\mathrm{t}, J=2.4 \mathrm{~Hz}, 1 \mathrm{H}, \mathrm{ArCH}), 4.83-4.81\left(\mathrm{~m}, 2 \mathrm{H}, \mathrm{CH}_{2}\right), 3.83\left(\mathrm{~s}, 3 \mathrm{H}, \mathrm{OCH}_{3}\right), 2.12(\mathrm{~s}, 3 \mathrm{H}$, $\left.\mathrm{O}_{2} \mathrm{CCH}_{3}\right), 1.63(\mathrm{t}, J=3.0 \mathrm{~Hz}, 3 \mathrm{H}, \mathrm{CH} 3) .{ }^{13} \mathrm{C} \mathrm{NMR}\left(100 \mathrm{MHz}, \mathrm{CDCl}_{3}\right) \delta: 206.1(\mathrm{C}=\mathrm{C}=\mathrm{C}), 170.0$ $\left(\mathrm{CO}_{2}\right), 159.5\left(\mathrm{CH}_{3} \mathrm{O} C_{A r}\right), 130.4\left(\mathrm{CHC}_{A r}\right), 128.6\left(2 \times o-\mathrm{C}_{A r}\right), 113.8\left(2 \times m-\mathrm{C}_{A r}\right), 99.3\left(C=\mathrm{C}=\mathrm{CH}_{2}\right), 76.7$ $\left(\mathrm{CH}_{2}\right), 75.6(\mathrm{CH}), 55.3\left(\mathrm{OCH}_{3}\right), 21.2\left(\mathrm{O}_{2} \mathrm{CCH}_{3}\right), 15.1\left(\mathrm{CH}_{3}\right)$. IR (neat, $\left.\mathrm{cm}^{-1}\right) 2934(\mathrm{br}), 2837(\mathrm{w}), 1960$ (w), 1736 (s), 1512 (m), 1369 (m), 1226 (s), 1172 (s), 838 (s). HRMS (ESI) calcd for $\mathrm{C}_{14} \mathrm{H}_{20} \mathrm{O}_{3} \mathrm{~N}$ $\left[\mathrm{M}+\mathrm{NH}_{4}\right]^{+} 250.1438$, obsd 250.1434 .

\section{General procedure for the preparation of iodo-compounds 13 and 14:}

The gold catalyst 1 ( $4.6 \mathrm{mg}, 0.01 \mathrm{mmol}, 0.05$ equiv.) in DCM $(0.60 \mathrm{~mL})$ was left at rt without stirring for $5 \mathrm{~min}$. Allene 12a or 12b (0.12 mmol), NIS (33.0 mg, $0.15 \mathrm{mmol}, 1.25$ equiv.) and water (0.06 $\mathrm{mL}, 5$ vol\%) in DCM $(0.60 \mathrm{~mL})$ were added and the solution was stirred for 2 hrs at rt. The mixture was filtered through $\mathrm{Na}_{2} \mathrm{SO}_{4}$ and concentrated. Flash chromatography (n-pentane/EtOAc 10:1) afforded products 13 and 14.

\section{2-Hydroxy-3-iodo-1-(4-methoxyphenyl)-2-methylbut-3-en-1-yl benzoate (13)}

The title compound was prepared from allene 12a and NIS in DCM in the presence of gold catalyst $\mathbf{1}$ as described in the General procedure above, affording the product as an orange oil (31.2 $\mathrm{mg}, 59 \%)$, 
$\mathrm{R}_{f}=0.65$ (n-hexane/EtOAc 4:1). ${ }^{1} \mathrm{H}$ NMR (400 MHz, $\left.\mathrm{CDCl}_{3}\right) \delta: 8.07-8.05\left(\mathrm{~m}, 2 \mathrm{H}, \mathrm{H}_{A r}\right), 7.60-7.55$ $\left(\mathrm{m}, 1 \mathrm{H}, \mathrm{H}_{A r}\right), 7.49-7.46\left(\mathrm{~m}, 2 \mathrm{H}, \mathrm{H}_{A r}\right), 7.44-7.40\left(\mathrm{~m}, 2 \mathrm{H}, \mathrm{H}_{A r}\right), 6.89-6.86\left(\mathrm{~m}, 2 \mathrm{H}, \mathrm{H}_{A r}\right), 6.47(\mathrm{~d}, J=$ $\left.2.2 \mathrm{~Hz}, 1 \mathrm{H}, \mathrm{CH} H_{a}\right), 6.27(\mathrm{~s}, 1 \mathrm{H}, \mathrm{ArCH}), 5.97\left(\mathrm{~d}, J=2.2 \mathrm{~Hz}, 1 \mathrm{H}, \mathrm{CH} H_{b} \mathrm{H}\right), 3.79\left(\mathrm{~s}, 3 \mathrm{H}, \mathrm{OCH}_{3}\right), 2.45$ (s, $1 \mathrm{H}, \mathrm{OH}), 1.37\left(\mathrm{~s}, 3 \mathrm{H}, \mathrm{CH}_{3}\right) \cdot{ }^{13} \mathrm{C} \mathrm{NMR}\left(100 \mathrm{MHz}, \mathrm{CDCl}_{3}\right) \delta: 165.3\left(\mathrm{CO}_{2}\right), 159.7\left(\mathrm{CH}_{3} \mathrm{OC}_{A r}\right)$, $133.2\left(p-\mathrm{C}_{A r}\right), 130.0\left(\mathrm{O}_{2} \mathrm{C} C_{A r}\right), 129.7\left(2 \times o-\mathrm{C}_{A r}\right), 129.5\left(2 \times o-\mathrm{C}_{A r}\right), 128.5\left(2 \times m-\mathrm{C}_{A r}\right), 128.0\left(\mathrm{CHC}_{A r}\right)$, 127.1 $\left(\mathrm{CH}_{2}\right), 117.6(\mathrm{CI}), 113.5\left(2 \times m-\mathrm{C}_{A r}\right), 78.9(\mathrm{CH}), 78.5(\mathrm{COH}), 55.2\left(\mathrm{OCH}_{3}\right), 23.8\left(\mathrm{CH}_{3}\right) . \mathrm{IR}$ (neat, $\mathrm{cm}^{-1}$ ) 3494 (br), 2926 (br), 2844 (w), 1704 (m), 1611 (m), 1512 (m), 1245 (s), 1025 (s), 709 (s); HRMS (ESI) calcd for $\mathrm{C}_{19} \mathrm{H}_{23} \mathrm{O}_{4} \mathrm{NI}\left[\mathrm{M}+\mathrm{NH}_{4}\right]^{+}$456.0666; obsd 456.0667 .

\section{3-Iodo-2-(4-methoxyphenyl)-2-methylbut-3-enal (14)}

The title compound was prepared according to the General procedure above from gold catalyst $\mathbf{1}$ (6.6 $\mathrm{mg}, 0.01 \mathrm{mmol}, 0.05$ equiv.) in DCM (0.70 mL), allene 12b (40.0 mg, $0.17 \mathrm{mmol})$, NIS (48.0 mg, $0.21 \mathrm{mmol}, 1.25$ equiv. $)$ in water $(0.09 \mathrm{~mL}, 5 \mathrm{vol} \%)$ and DCM $(1.00 \mathrm{~mL})$ as a yellow oil $(24.2 \mathrm{mg}$, 45\%), $\mathrm{R}_{f}=0.42$ (n-hexane/EtOAc 4:1). ${ }^{1} \mathrm{H}$ NMR (400 MHz, $\left.\mathrm{CDCl}_{3}\right) \delta: 9.76$ (s, $\left.1 \mathrm{H}, \mathrm{OCH}\right), 7.24-7.21$ $\left(\mathrm{m}, 2 \mathrm{H}, \mathrm{H}_{A r}\right), 6.97-6.94\left(\mathrm{~m}, 2 \mathrm{H}, \mathrm{H}_{A r}\right), 6.26\left(\mathrm{~d}, J=2.8 \mathrm{~Hz}, 1 \mathrm{H}, \mathrm{CH}_{\mathrm{a}} \mathrm{H}\right), 6.18(\mathrm{~d}, J=2.8 \mathrm{~Hz}, 1 \mathrm{H}$, $\left.\mathrm{CH}_{\mathrm{b}}\right), 3.84\left(\mathrm{~s}, 3 \mathrm{H}, \mathrm{OCH}_{3}\right), 1.67\left(\mathrm{~s}, 3 \mathrm{H}, \mathrm{CH}_{3}\right) .{ }^{13} \mathrm{C} \mathrm{NMR}\left(100 \mathrm{MHz}, \mathrm{CDCl}_{3}\right) \delta: 196.9(\mathrm{OCH}), 159.7$ $\left(\mathrm{CH}_{3} \mathrm{OC}_{A r}\right), 130.3\left(\mathrm{C}_{A r}\right), 129.0\left(2 \times o-\mathrm{C}_{A r}\right), 128.3\left(\mathrm{CH}_{2}\right), 115.6(\mathrm{CI}), 114.4\left(2 \times o-\mathrm{C}_{A r}\right), 62.7\left(\mathrm{CH}_{3} C\right)$, $55.3\left(\mathrm{CH}_{3} \mathrm{O}\right), 21.2\left(\mathrm{CH}_{3}\right)$, ). IR (neat, $\left.\mathrm{cm}^{-1}\right) 2935(\mathrm{br}), 2835(\mathrm{w}), 1723(\mathrm{~s}), 1605(\mathrm{~m}), 1509(\mathrm{~s}), 1250(\mathrm{~s})$ 1029 (s), 827 (s). HRMS (ESI) calcd for $\mathrm{C}_{12} \mathrm{H}_{17} \mathrm{O}_{2} \mathrm{NI}\left[\mathrm{M}+\mathrm{NH}_{4}\right]^{+} 334.0298$, obsd 334.0298.

\section{Reference}

1. Aaseng, J. E.; Iqbal, N.; Sperger, C. A.; Fiksdahl, A. 2014, submitted.

2. Bae, H J.; Jeong, W.; Lee, J. H.; Rhee, Y. H. Chem.-- Eur. J. 2011, 17, 1433. 
${ }^{1} \mathrm{H}$ and ${ }^{13} \mathrm{C}$-spektra of compounds $7 \mathrm{a}, 7 \mathrm{~b}, 8,9,10,11,12 \mathrm{a}, 12 \mathrm{~b}, 13$ and 14

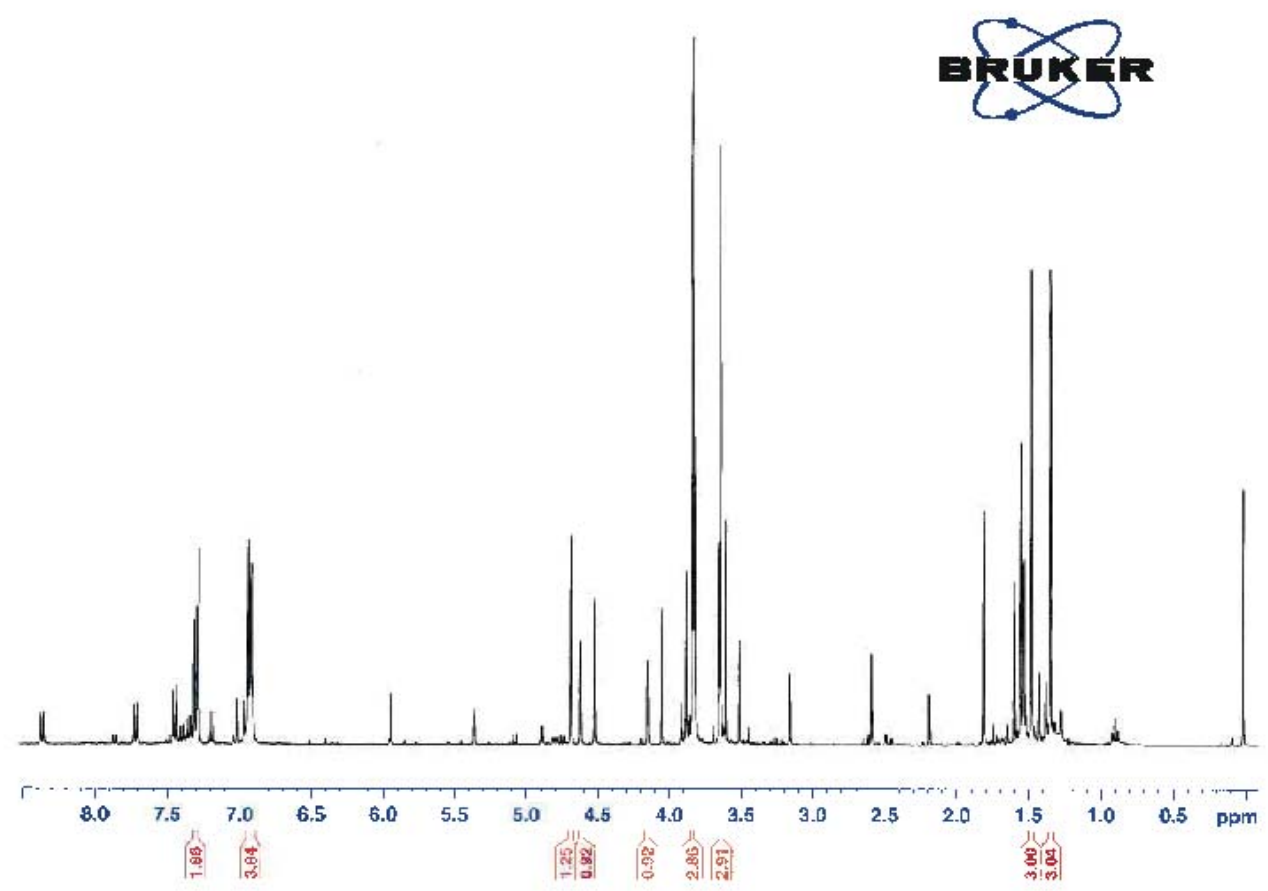

Fig. $1{ }^{1} \mathrm{H}$ NMR spectrum of $7 \mathbf{a}$ in $\mathrm{CDCl}_{3}$

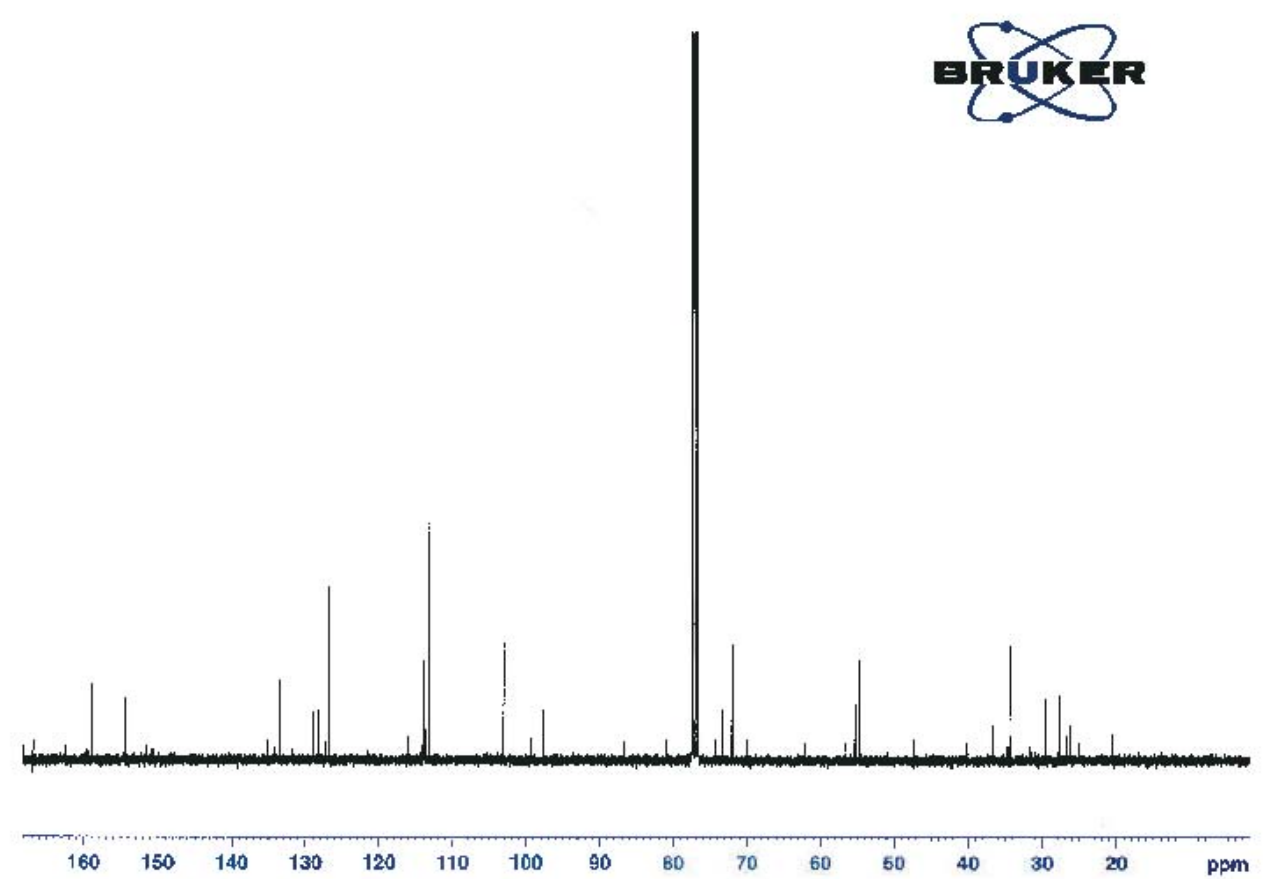

Fig. $2{ }^{13} \mathrm{C}$ NMR spectrum of $7 \mathbf{a}$ in $\mathrm{CDCl}_{3}$ 


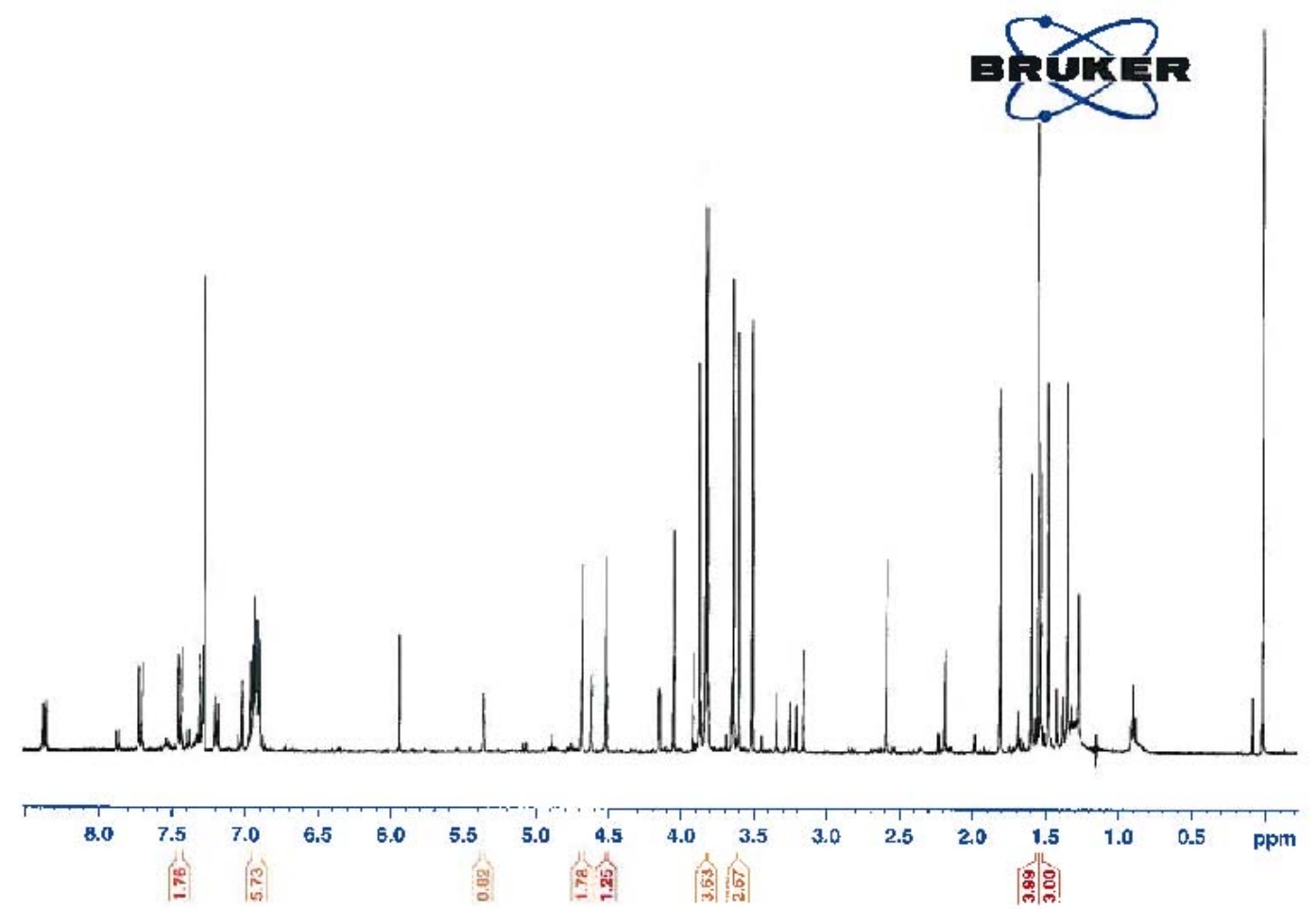

Fig. $3{ }^{1} \mathrm{H}$ NMR spectrum of $\mathbf{7 b}$ in $\mathrm{CDCl}_{3}$

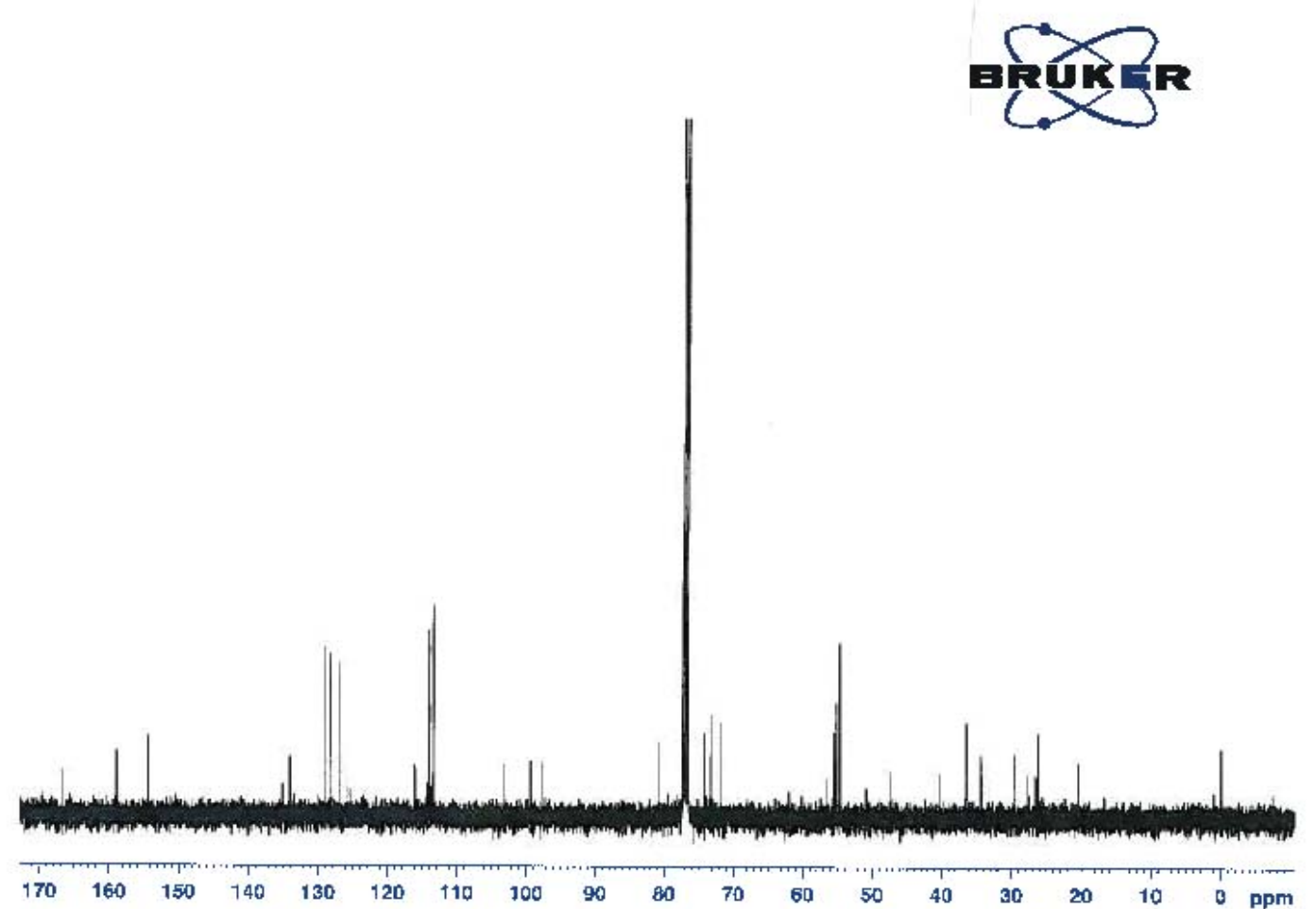

Fig. $4{ }^{13} \mathrm{C}$ NMR spectrum of $7 \mathbf{b}$ in $\mathrm{CDCl}_{3}$ 


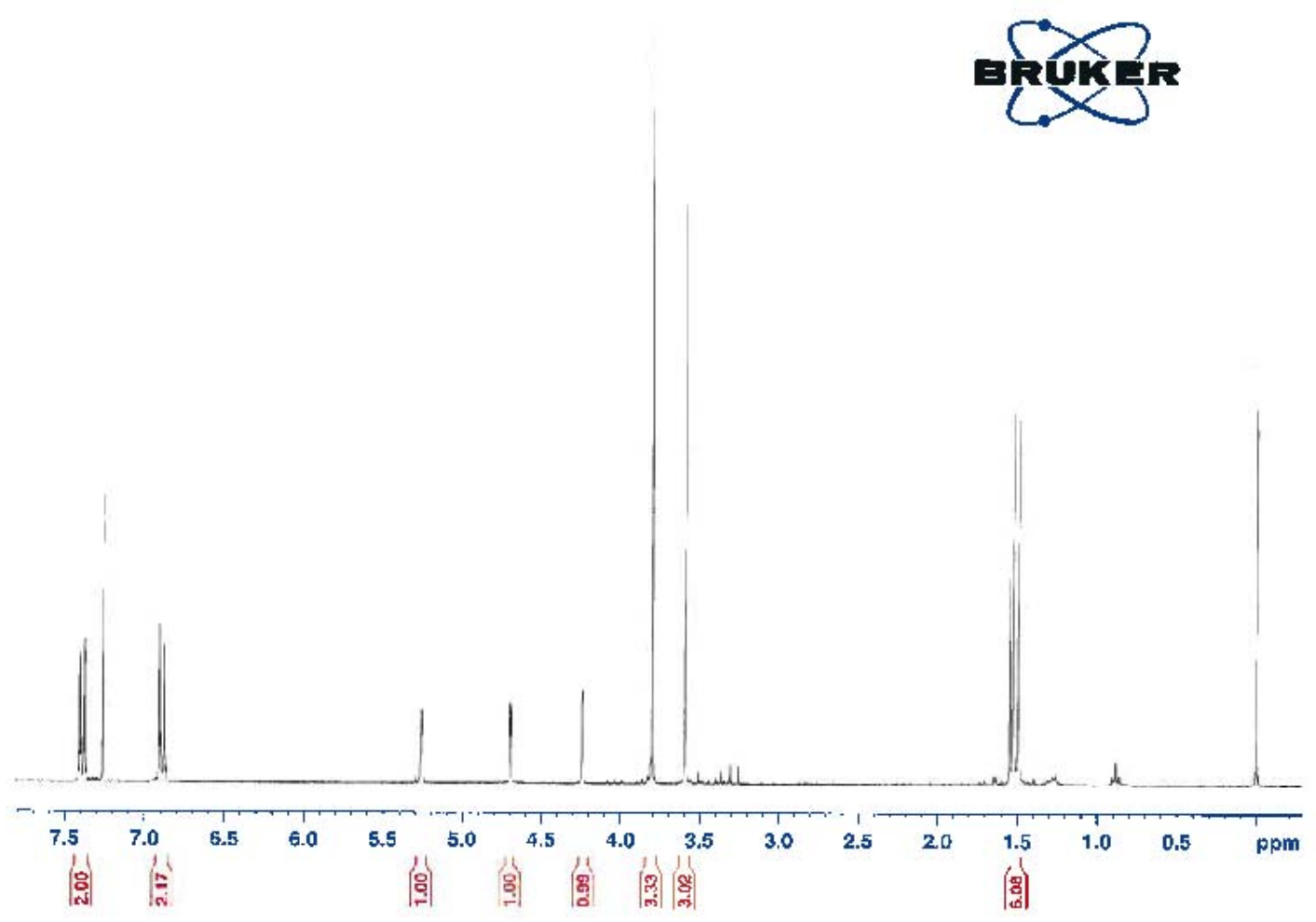

Fig. $5{ }^{1} \mathrm{H}$ NMR spectrum of 8 in $\mathrm{CDCl}_{3}$

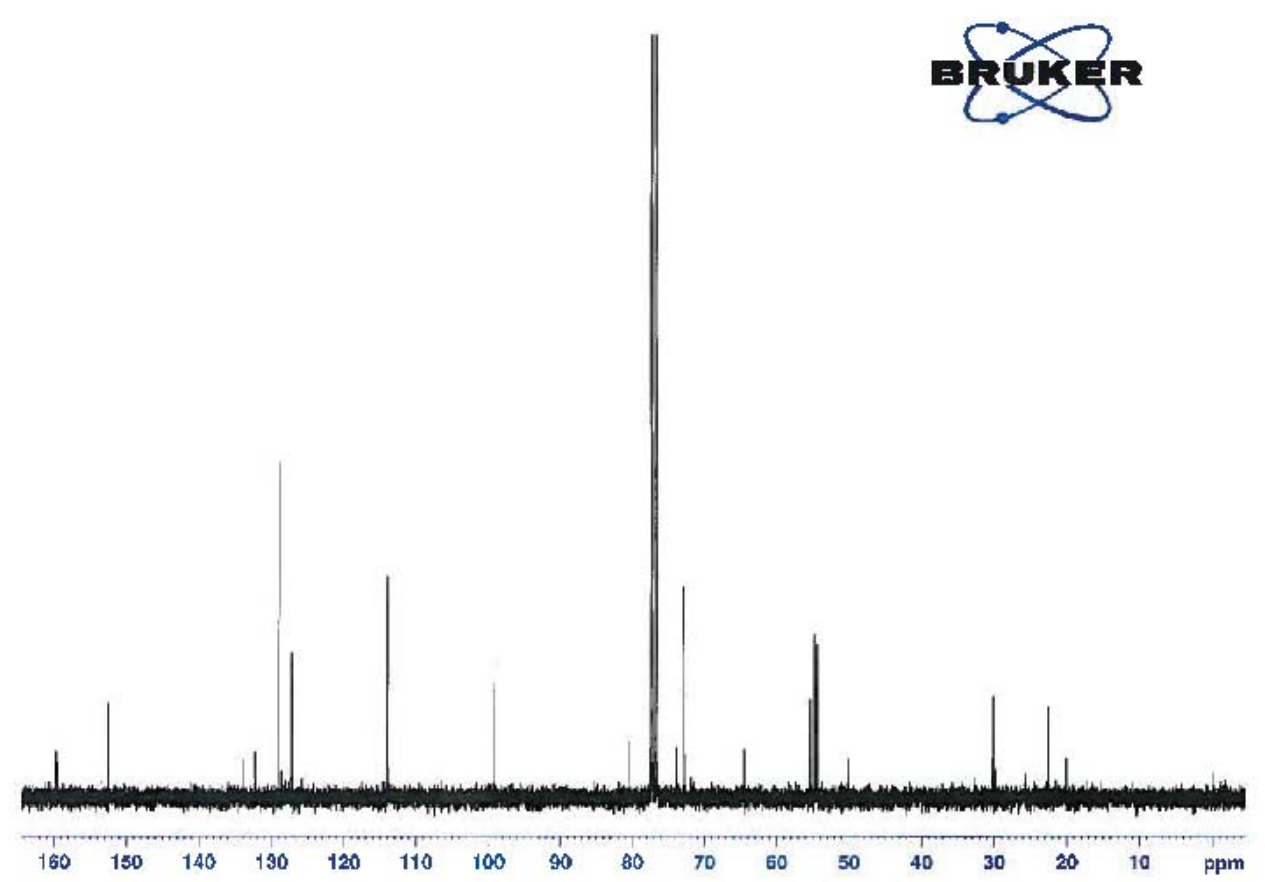

Fig. $6{ }^{13} \mathrm{C}$ NMR spectrum of 8 in $\mathrm{CDCl}_{3}$ 


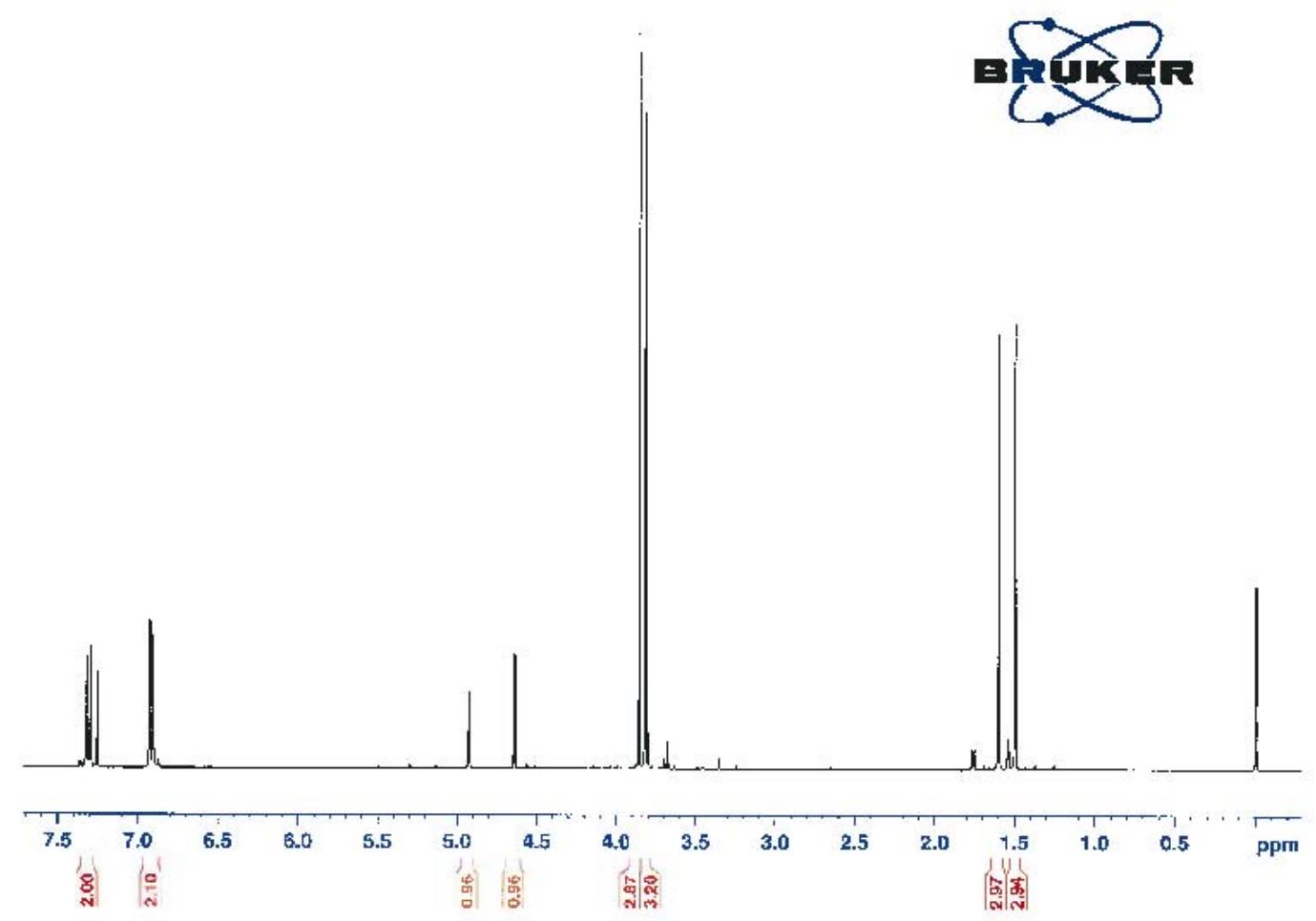

Fig. $7{ }^{1} \mathrm{H}$ NMR spectrum of 9 in $\mathrm{CDCl}_{3}$

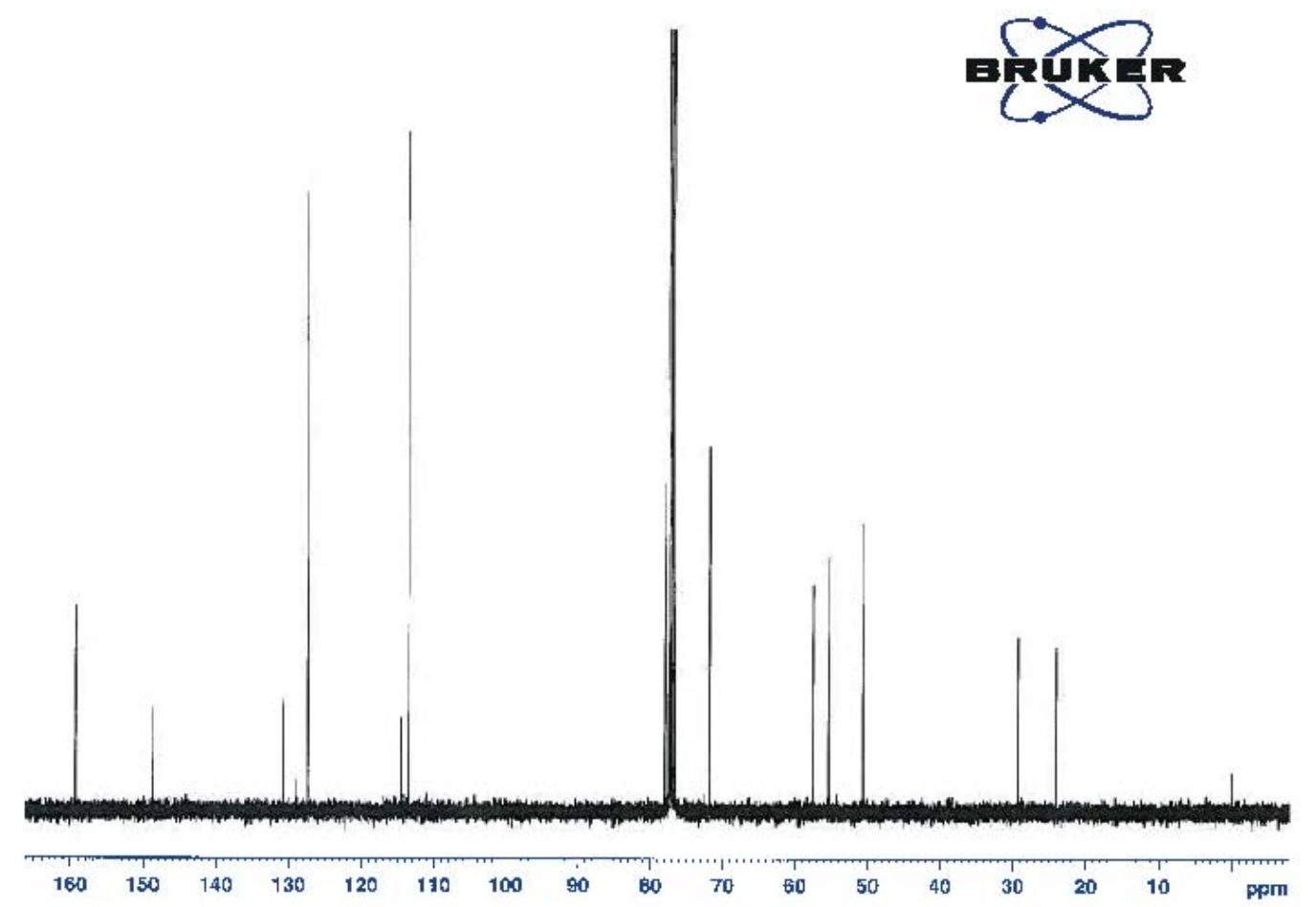

Fig. $8{ }^{13} \mathrm{C}$ NMR spectrum of 9 in $\mathrm{CDCl}_{3}$ 


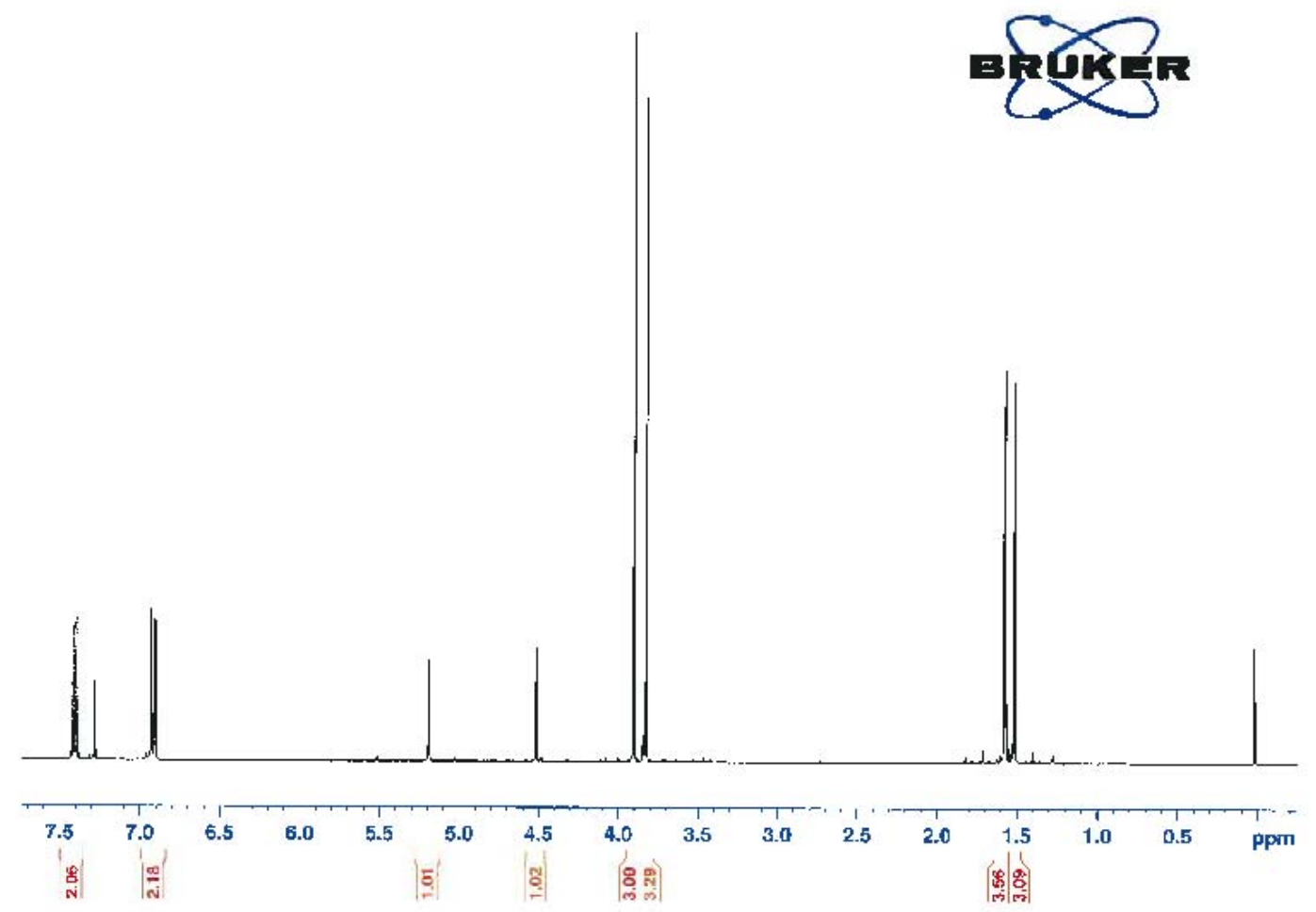

Fig. $9{ }^{1} \mathrm{H}$ NMR spectrum of $\mathbf{1 0}$ in $\mathrm{CDCl}_{3}$

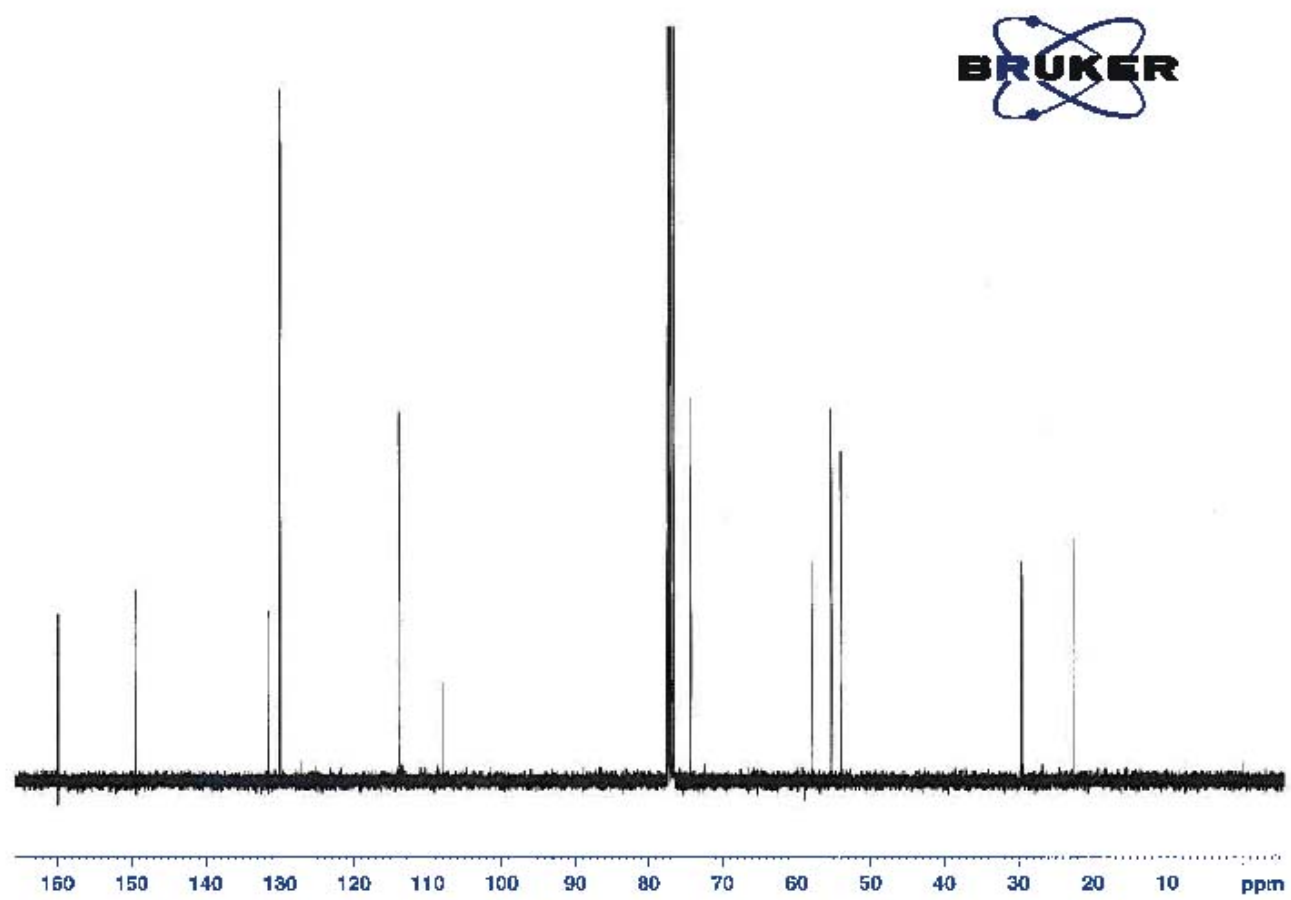

Fig. $10{ }^{13} \mathrm{C}$ NMR spectrum of $\mathbf{1 0}$ in $\mathrm{CDCl}_{3}$ 


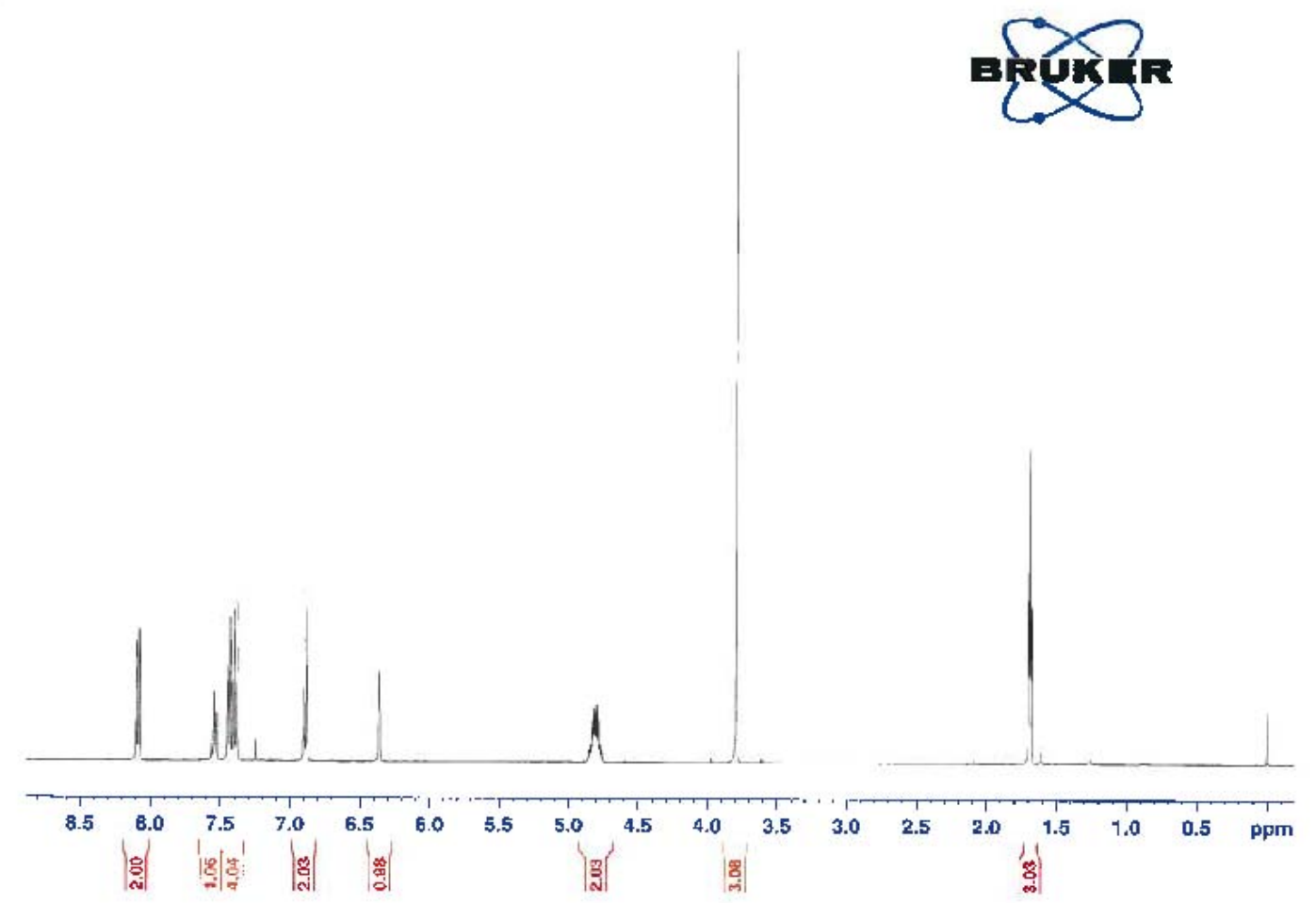

Fig. $11^{1} \mathrm{H}$ NMR spectrum of 12a in $\mathrm{CDCl}_{3}$

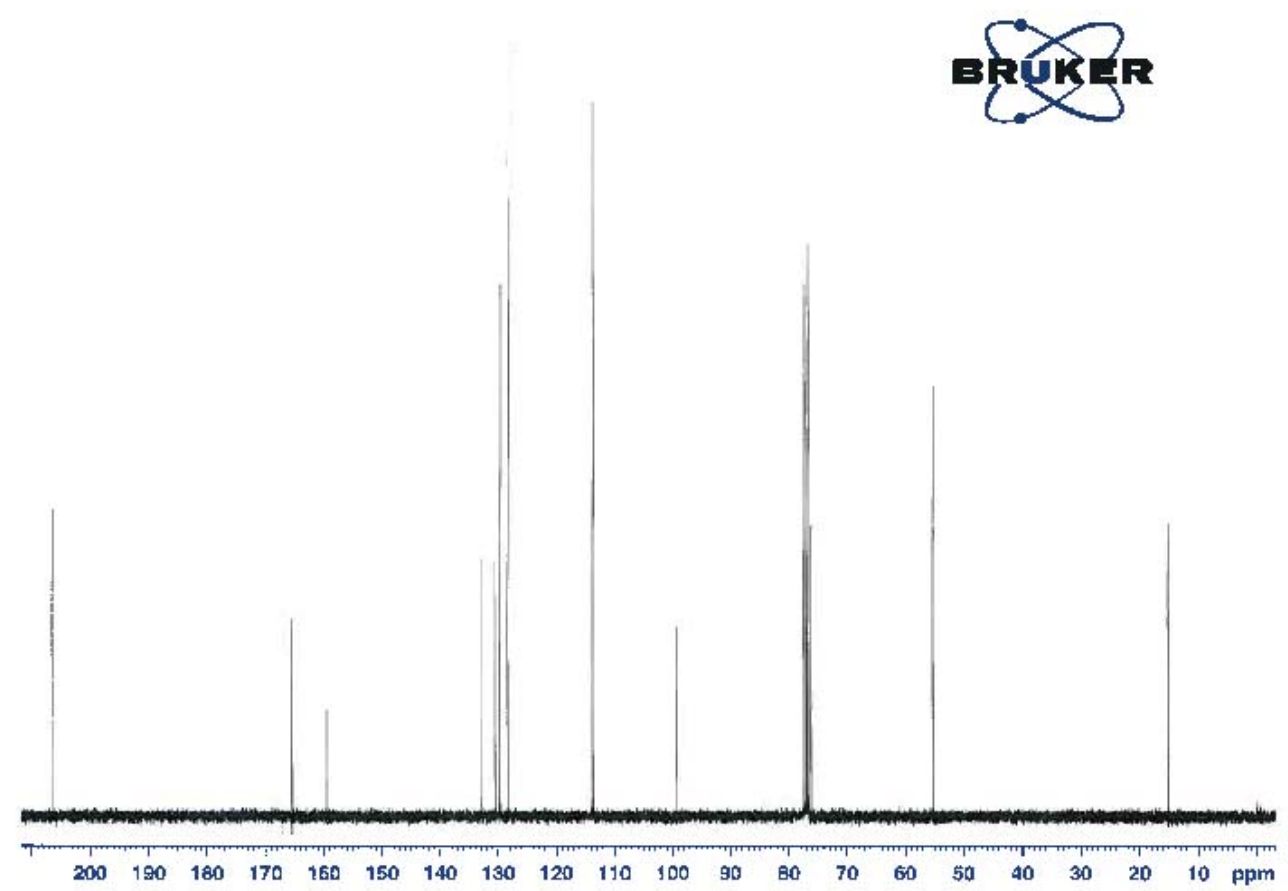

Fig. $12{ }^{13} \mathrm{C}$ NMR spectrum of 12a in $\mathrm{CDCl}_{3}$ 


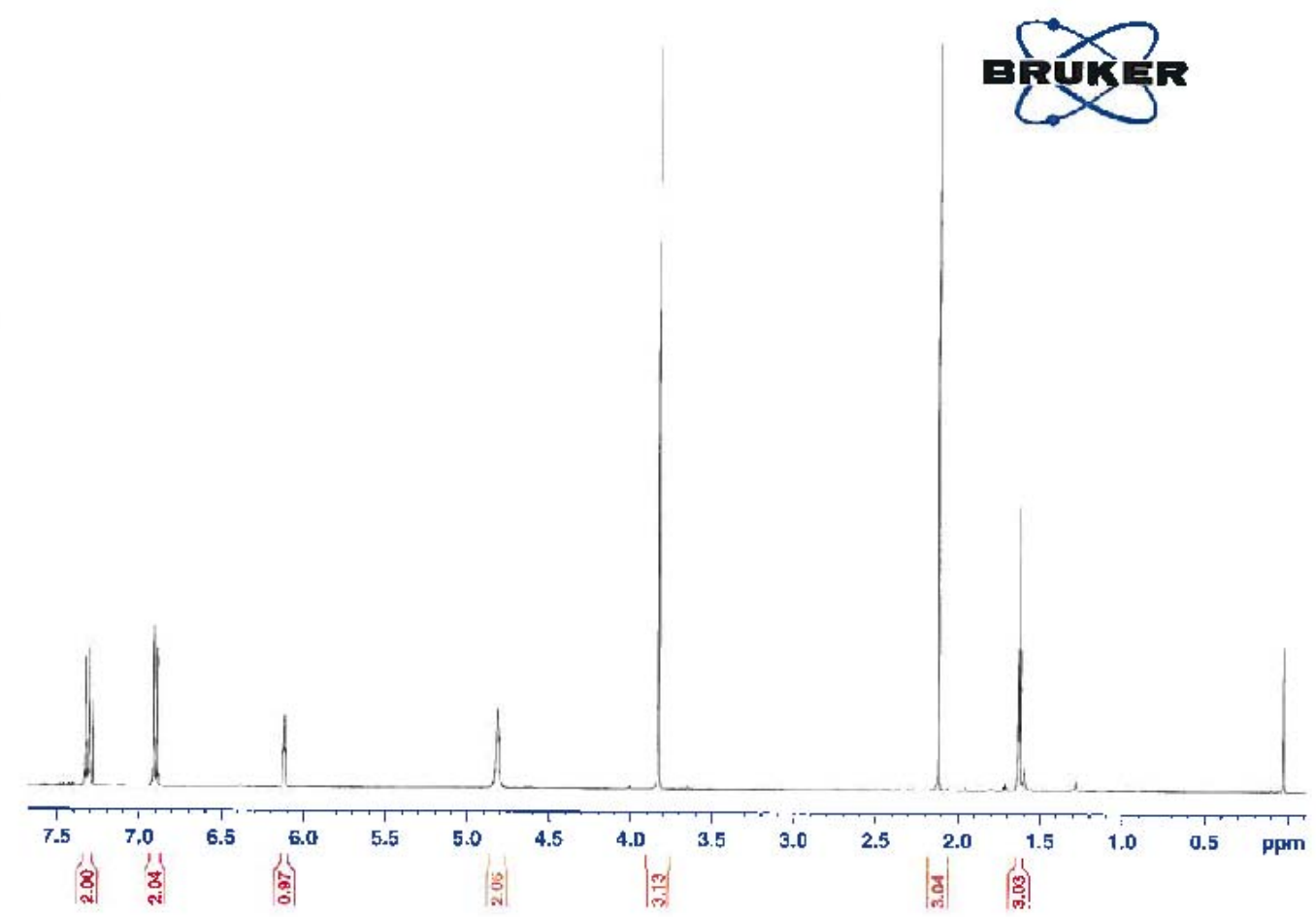

Fig. $13{ }^{1} \mathrm{H}$ NMR spectrum of $\mathbf{1 2 b}$ in $\mathrm{CDCl}_{3}$

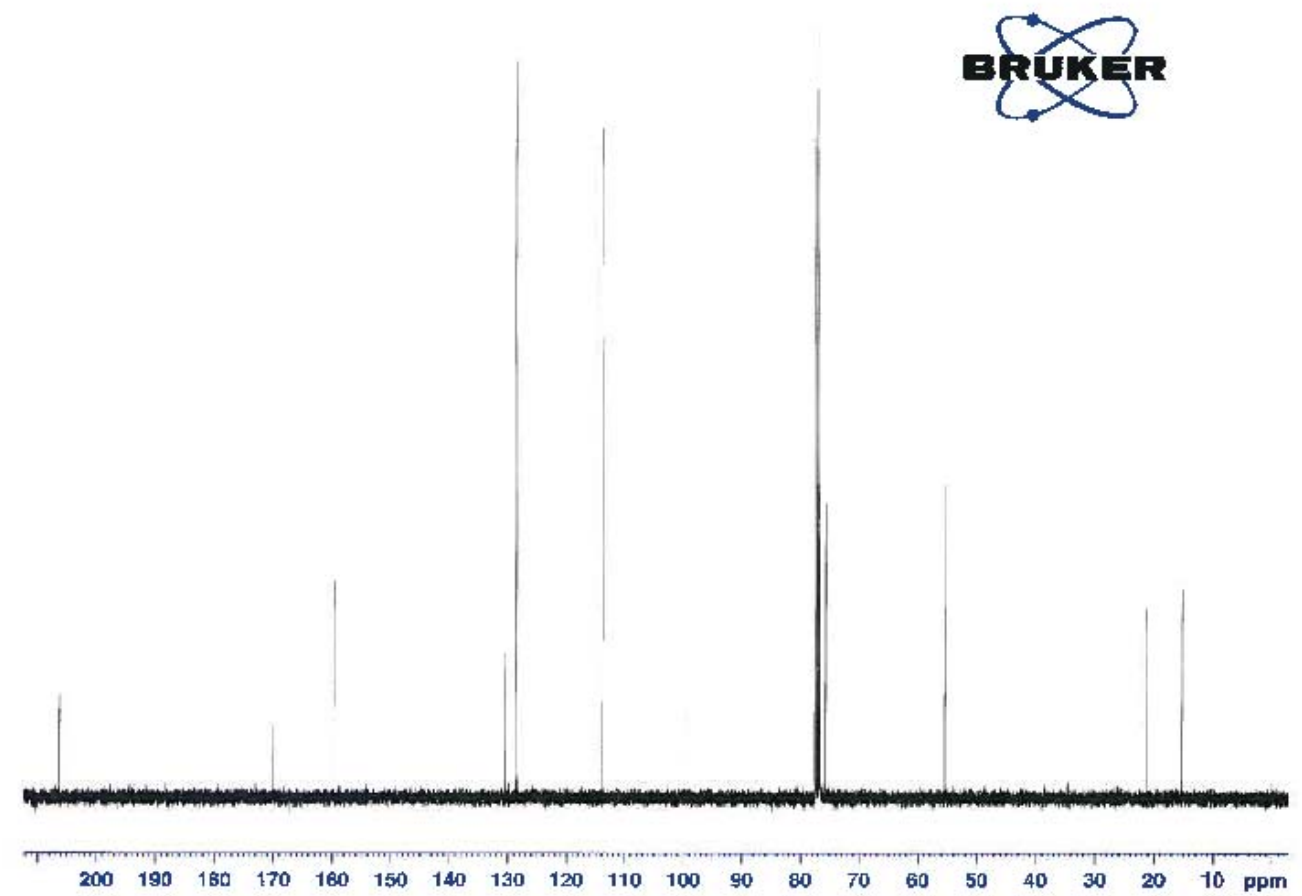

Fig. $14{ }^{13} \mathrm{C}$ NMR spectrum of $\mathbf{1 2 b}$ in $\mathrm{CDCl}_{3}$ 


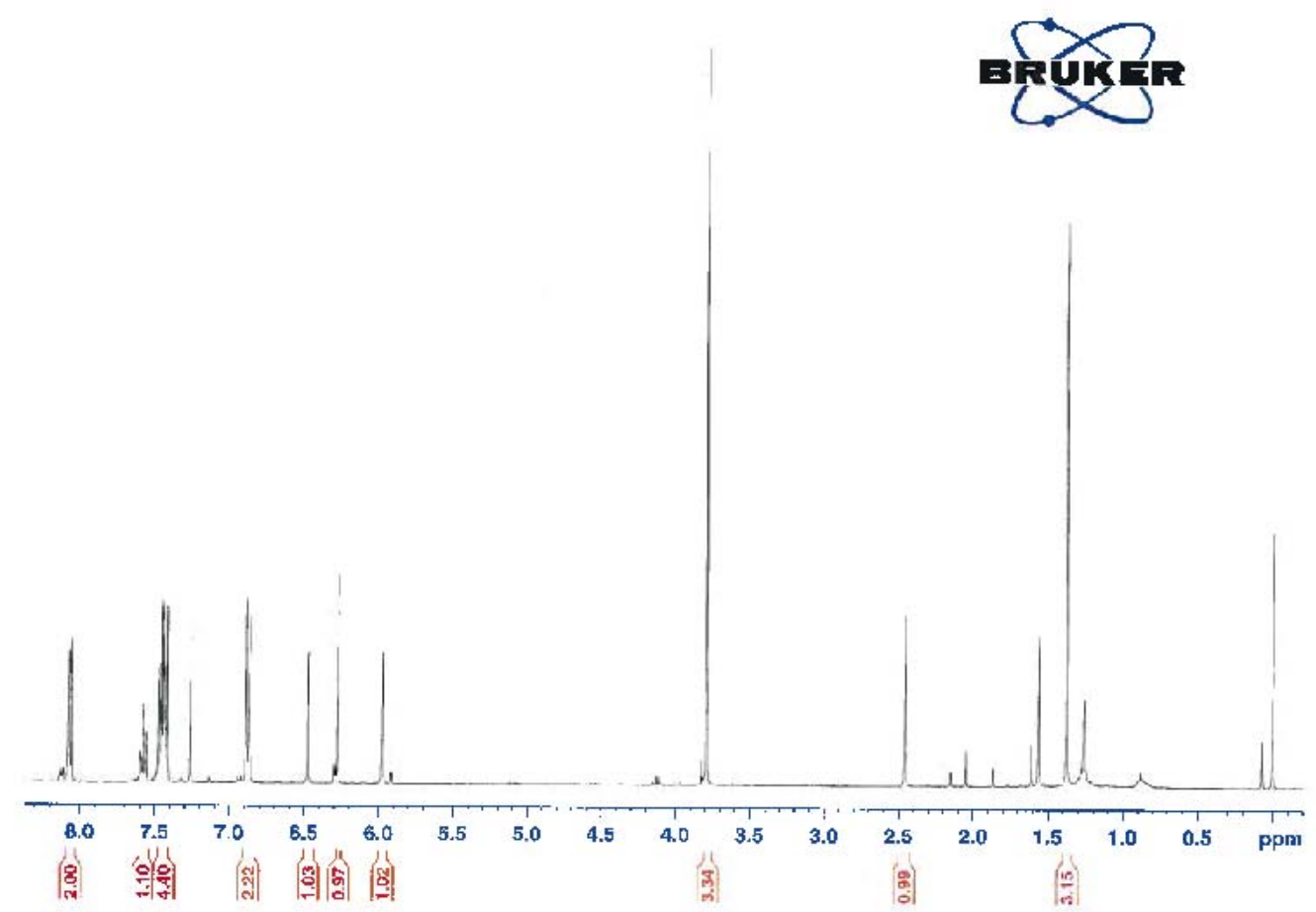

Fig. $15{ }^{1} \mathrm{H}$ NMR spectrum of 13 in $\mathrm{CDCl}_{3}$

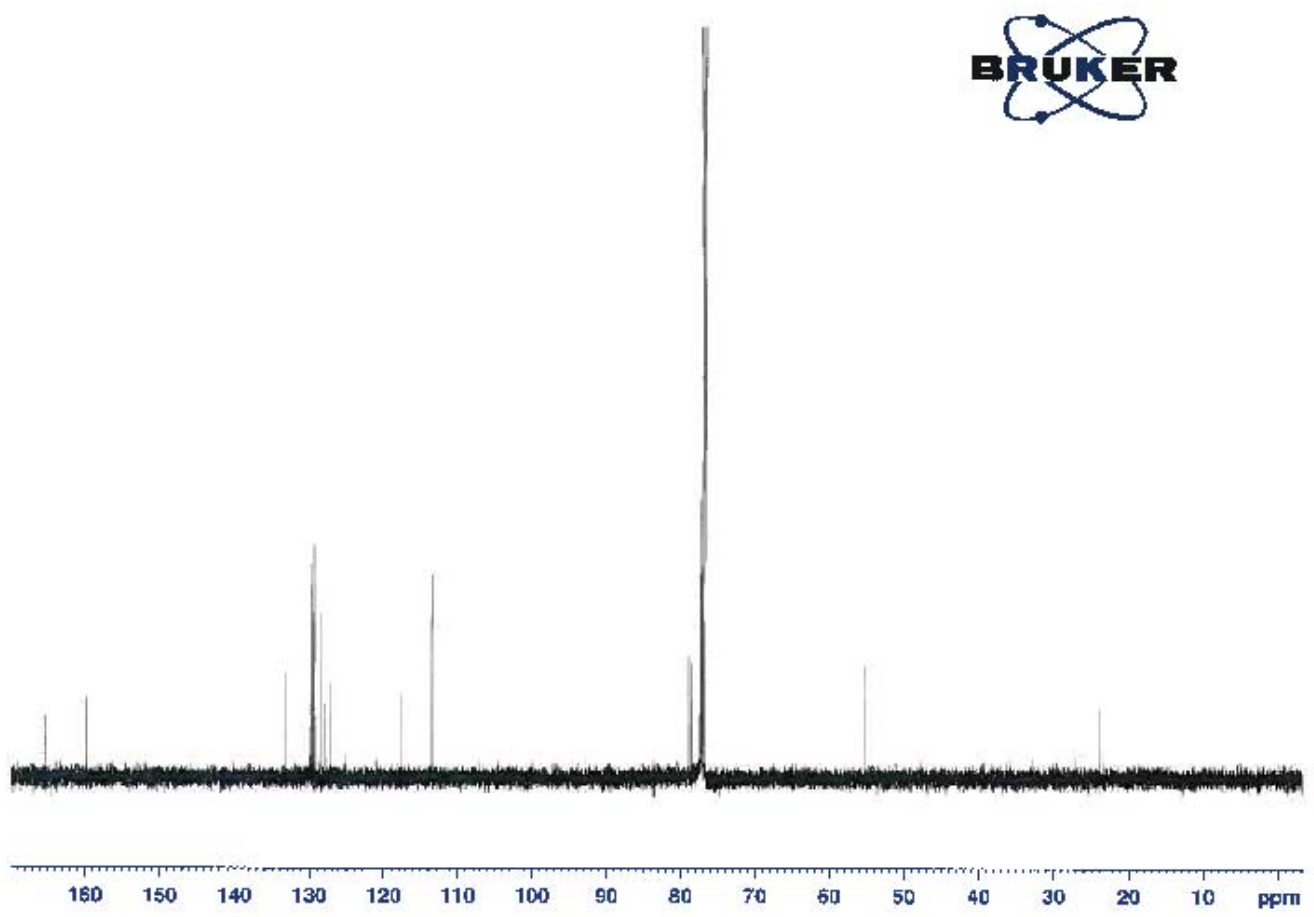

Fig. $16{ }^{13} \mathrm{C}$ NMR spectrum of 13 in $\mathrm{CDCl}_{3}$ 


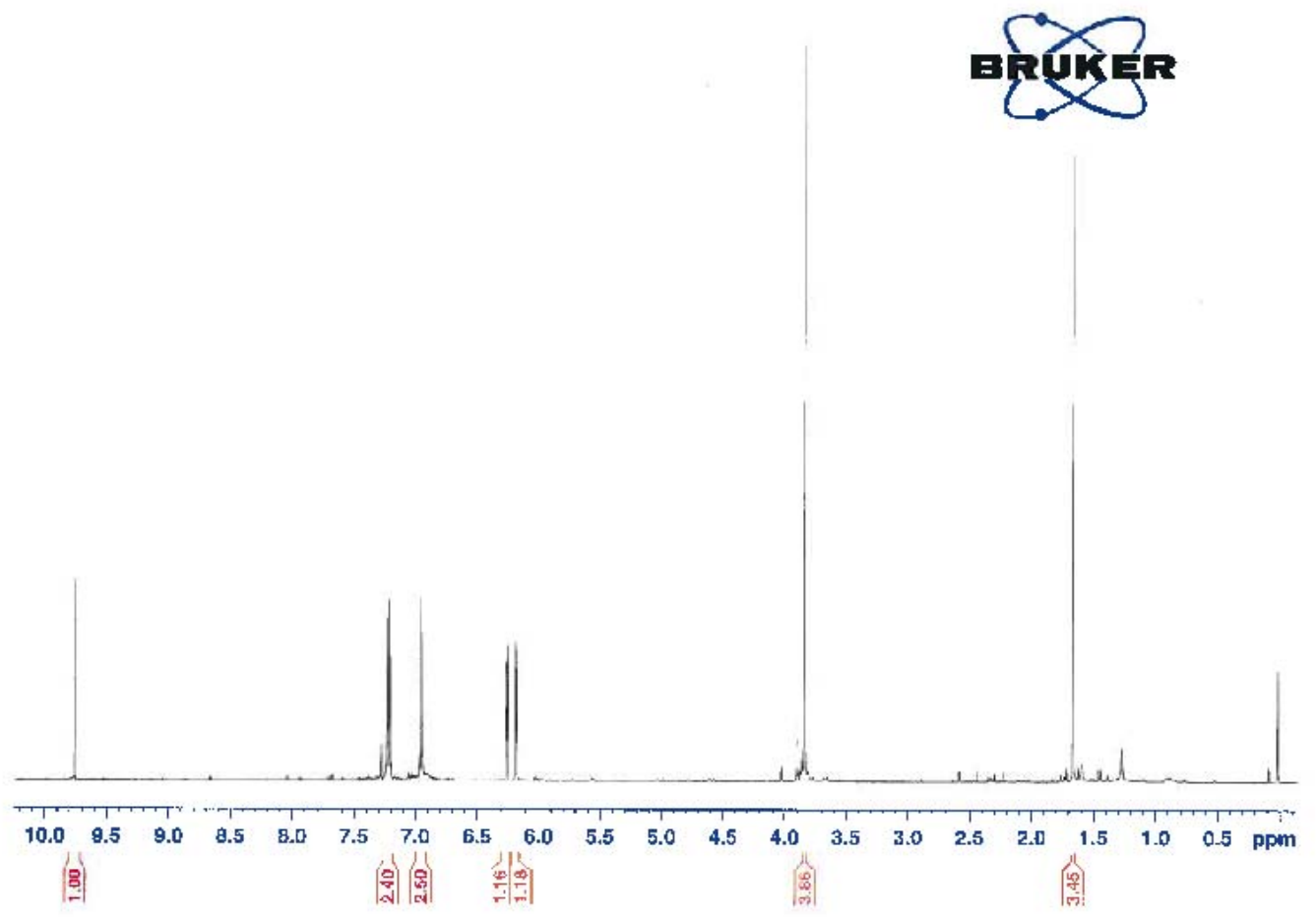

Fig. $17^{1} \mathrm{H}$ NMR spectrum of 14 in $\mathrm{CDCl}_{3}$

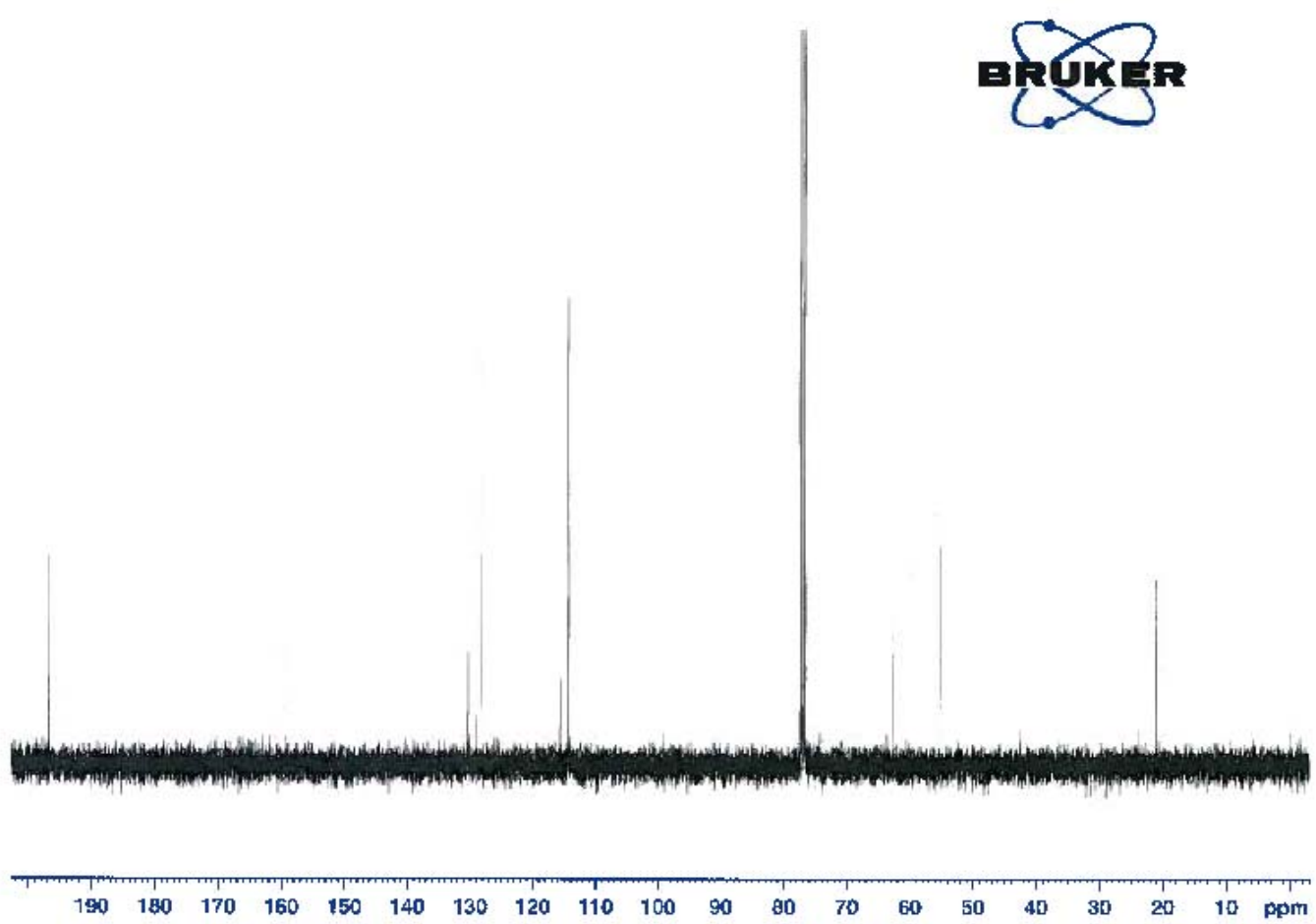

Fig. $18{ }^{13} \mathrm{C}$ NMR spectrum of 14 in $\mathrm{CDCl}_{3}$ 